\title{
Intervención breve en Cantabria en problemas relacionados con el alcohol
}

\author{
Díez, J.F.; Peña, C.; García, E.; Gaite, L. \\ Servicio de Psiquiatría. Hospital Universitario "Marqués de Valdecilla". Santander CANTABRIA
}

Enviar correspondencia a:

Prof. J.F. Déz Manrique. Servicio de Psiquiatría. Hospital Universitario “Marqués de Valdecilla". Avda. Valdecilla s/n. 39008 Santander, CANTABRIA.

\section{RESUMEN}

Los modelos de intervención breve han demostrado ser un tratamiento eficaz para disminuir el consumo de etanol en personas bebedoras de riesgo, con bajo coste y desde contextos no especializados como la Atención Primaria.

En este trabajo se presentan parte de los resultados de una linea de investigación que manejaba actuaciones de tipo informativo-educativo con la finalidad de llevar a a sujetos bebedores de riesgo, no alcohol dependientes, hacia consumos más moderados.

Participaron en el estudio 1022 varones, entre 18 y 65 años de edad, bebedores habituales entre 21 y 95 unidades semanales, que fueron evaluados mediante autoinforme detallado de su consumo en la semana anterior a la entrevista y seis meses después de la intervención. En cuatro contextos sociosanitarios distintos, los sujetos fueron aleatorizados en grupos de simple evaluación y grupos de intervención, consistente ésta en la entrega y explicación de un folleto de autoayuda asociado a la misma sesión de evaluación.

Los resultados obtenidos muestran tendencias significativas a disminuir el consumo inicial en la mayoría de parámetros de cambio utilizados, así como efectos aparentemente paradójicos, descenso en el consumo de los grupos de simple evaluación.

Palabras Clave. Tratamiento, intervención breve, consumo de riesgo, alcoholismo.

\section{SUMMARY}

It has been demonstrated that brief intervention models are effective in reducing ethanol consumption among primary care patients at-risk drinkers, with a low economic cost. The purpose of this study was to evaluate the effects of informativeeducational brief interventions in non-dependent alcohol drinkers. A substantial reduction in alcohol consumption was expected. The subjects were 1022 males (range of age 18-65 years) who usually drink 21-95 international unit per week. After screening for eligibility, the participants completed a baseline questionnaire on alcohol consumption during the previous week to the interview. In four different health care settings, subjects were randomly assigned to the informativeeducational brief intervention condition or to standard evaluation. The informative-educational intervention included a self-help booklet that was given to all participants during the first evaluation interview. Alcohol consumption patterns were re-evaluated in six months. The results show, a significant reduction in the baseline alcohol consumption and some other paradoxical effects, drinker reduction in standard evaluation groups.

Key words: treatment; brief intervention; at-risk alcohol; alcoholism.

\section{INTRODUCCIÓN}

I consumo de bebidas alcohólicas, y las repercu- siones de él derivadas, es considerado como uno de los problemas sociosanitarios más severos con que se enfrentan las sociedades occidentales. Los esfuerzos realizados en estos países, dentro del ámbito de las políticas sanitarias, han ido encaminados, hasta hace relativamente poco tiempo, al trata- miento de aquellas personas que en un momento dado presentan, con mayor o menor intensidad, los problemas conceptualizados con el término "alcoholismo", considerado éste como un severo factor de riesgo de un sinfín de patologías. Estos trastornos afectan a todo el funcionamiento biopsicosocial del individuo y suelen incluirse en el amplio concepto de los problemas relacionados con el consumo de alcohol (PRA). Desde el entorno de la OMS se han realizado intentos de clasificar y agrupar los PRA (Miller, 
Sovereign, Nathan y Marlatt, 1989; Moser, 1989) y entre nosotros Gutiérrez (1993) ha realizado una exhaustiva revisión de la presencia de esos problemas en la comunidad.

En nuestro medio hemos podido constatar, en la población adulta de ambos sexos, unas prevalencias de consumo excesivo, propias de personas alcoholdependientes ((100 c.c. del alcohol puro/día, equivalente a 80 gr. o 10 unidades), del 7,3\% en toda la comunidad autónoma, y del 5,1\% si nos circunscribimos a la ciudad de Santander (Diez Manrique, 1991; Peña Martín, 1995). Se describían como abstemios un $16 \%$ de la población de la comunidad autónoma y un $27 \%$ en el entorno urbano, mientras que el $70 \%$ de los varones y el $31 \%$ de las mujeres mayores de 16 años, eran consumidores habituales de bebidas alcohólicas al menos dos/tres días a la semana.

Una linea de programas, propiciada desde la OMS, como el "Estudio de la Respuesta Comunitaria a los Problemas Relacionados con el Consumo de Alcohol (PRA)" (Ritson, 1985), constituyen una muestra del creciente interés por centrar los esfuerzos asistenciales en torno a aquellas personas, bebedoras habituales de cantidades moderadas o altas, que no presentan y posiblemente no llegaran nunca a presentar problemas importantes por su consumo de alcohol, pero que sí tienen comportamientos de riesgo y es probable que empiezan a aparecer PRA, aunque no los identifiquen como derivados de su consumo de bebidas alcohólicas.

En el ámbito de la Atención Primaria suele aceptarse como "umbral de riesgo", sugerente de un consumo posiblemente abusivo, un patrón de bebida cotidiano, o concentrado en 3/4 días a la semana, de alrededor de 280 grs. para el hombre y 168 grs. para la mujer. Ya a estos niveles es previsible que el bebedor presente algunos problemas físicos y, en especial, personales y de relación.

Independientemente del tipo de tratamiento, suele aceptarse que solo un abordaje especializado puede enfrentar en todas sus dimensiones la problemática presente en el bebedor alcohol-dependiente especialmente en estados crónicos (Rodríguez Martos, 1989). A otros niveles no especializados, como la Atención Primaria, les correspondería la actuación en todos aquellos otros casos de bebedores moderados o de riesgo con actuaciones de prevención primaria o secundaria encaminadas a mantener el buen control de consumo en el primer caso y a lograr pautas más moderadas en el segundo, sin olvidar las actuaciones encaminadas a motivar el inicio de un abordaje espe- cializado o mantener el control en sujetos bajo tratamiento.

Los modelos de Intervención Breve (IB) han sido la propuesta más habitual , entre los distintos grupos de trabajo, a efectos de actuar con objetivos de prevención secundaria, identificación temprana del problema y minimización del posible daño; de tal forma que ha dado lugar a investigaciones tan ambiciosas como lo auspiciado en el marco de la OMS como proyecto multinacional para la identificación y manejo de los problemas relacionados con el consumo de alcohol realizado simultáneamente en 10 países con un seguimiento de 1665 sujetos que cumplían los criterios de inclusión (Babor y Grant, 1992)

Se trata de actuaciones destinadas a sujetos bebedores de riesgo no alcohol-dependientes, poco costosas, cortas en la dedicación de tiempo (en torno a 10-15 minutos), de consejo, realizadas por un no especialista, con una función básicamente informativo-educativa y pretendidamente integrables en la practica clínica cotidiana, especialmente en el ámbito de la atención primaria. La IB efectiva es algo más que una practica de simple consejo más/menos inespecífico. En general se ha focalizado en aumentar la conciencia del problema mediante una adecuada identificación e información y aconsejar estrategias especificas de cambio adaptadas al sujeto.

Pretendemos exponer en este trabajo una parte de la línea de investigación desarrollada por el equipo del Servicio-Cátedra de Psiquiatría del Hospital Universitario "Marqués de Valdecilla" (Santander) manejando actuaciones de tipo informativo-educativo sobre varones bebedores moderados o de riesgo no alcoholdependientes. Presentamos los resultados de una investigación que evalúa la eficacia de la intervención breve versus la simple evaluación (IB/SE) en relación con el consumo de bebedores moderados y para llevar a sujetos bebedores de riesgo hacia consumos más moderados. También pretendemos evaluar los posibles efectos diferenciales asociados a distintos contextos sociosanitarios.

\section{MATERIAL Y MÉTODOS}

Para poner en práctica la intervención se seleccionaron cuatro contextos distintos mediante los correspondientes convenios de colaboración con los equipos médicos de la zona. Se abordó solamente la problemática en población masculina. Los proyectos se iniciaron en su fase de campo a principios de 1990

\footnotetext{
'. Los datos que se presentan en este trabajo son parte de una investigación más amplia, cuyo proyecto y resultados fueron elaborados como Memoria de Actividad del Servicio-Catedra en el año 1998. La investigación fue parcialmente subvencionada por el Fonde de Investigaciones Sanitarias ( F.I.S ), Exp. Nº1/0689
} 
y el último de ellos cumplió su período de seguimiento de seis meses en octubre de 1994. Más en concreto se aplicó el procedimiento en:

- Hospital Universitario "Marqués de ValdeciIla": Se trabajó con pacientes que habían ingresado en el hospital por motivos médicos no psiquiátricos, excluyéndose aquellos que por el tipo de enfermedad o gravedad de la misma resultaba inaccesible su evaluación. En este sentido fueron excluidos, además de los psiquiátricos, pacientes con trastornos de conciencia, ACVA, diálisis, servicios como polivalentes, politraumatizados, oncología, etc.

- Medicina de Atención Primaria: Se utilizó como fuente de casos las consultas de un Centro de Salud de Atención Primaria de la ciudad de Santander, con características sociodemográficas muy específicas (Barrio Pesquero) y cuyo equipo médico estaba especialmente interesado en este tipo de problemas .

- Comunidad: Se escogió una pequeña comunidad rural (pueblo de Suesa en el municipio de Ribamontan al Mar) por las especiales condiciones del consumo en estos entornos, por ser una zona conocida por nosotros de estudios epidemiológicos previos, y por la especial accesibilidad del médico generalista titular de la misma.

- Medicina de Empresa: El interés del equipo médico de la empresa de productos químicos SOLVAY (Torrelavega) fue el motivo para incorporar el programa a su rutina de revisión médica anual a todos los trabajadores.

\section{Sujetos}

Fueron incorporados al estudio todos los sujetos, varones adultos entre 18 y 65 años (55 años como edad superior en la empresa por motivos administrativos de la misma) que una vez evaluados daban unas cifras de consumo habitual de bebidas alcohólicas igual o superior a 21 unidades semanales y menores de 95, al considerar que quienes superaran esa cifra podrían se encuadrados en la franja del alcoholismo y estar necesitados, en consecuencia, de otro tipo de intervenciones. Se decidió un mínimo muestral de sujetos que garantizase verificar tanto hipótesis de cambio en el promedio de consumo semanal (al menos 20 unidades), como descenso en la proporción de casos (un mínimo del $20 \%$ ) que en el pretratamiento tomaban cantidad de riesgo (35 u./semana o más), con un nivel de confianza del 95\%. Estimando por estudios previos en nuestro entorno el promedio, desviación standard, y proporción de sujetos en esa franja de consumo $(45,20$ y $60 \%$ de casos respectivamente) el tamaño de cada grupo muestral fue decidido mediante la fórmula (Carné, Moreno, Porta Serra y Velilla, 1989) $n=\left(2 S^{2} />^{2}\right) f$ donde "S" es la varianza estimada, ">" es la diferencia intergrupos esperada y "f" el error máximo admitido. Se utilizó un factor de corrección del tamaño muestral a fin de controlar la posible pérdida de casos (estimada en un 20\%) a lo largo del estudio. En torno a 100 sujetos/grupo parecieron necesarios para ajustarse a los requisitos anteriores.

Tabla 1. Número y edad de los sujetos

\begin{tabular}{|c|c|c|c|c|c|}
\hline & & & $\mathbf{M u}$ & & \\
\hline & & & & & \\
\hline & $N$ inicial & N & $\%$ inicial & Media & Rango \\
\hline Hospital & 364 & 283 & 77.7 & 44.4 & 13.9 \\
\hline Evaluación & 127 & 101 & 79.5 & 42.2 & 14.4 \\
\hline Intervención & 237 & 182 & 76.7 & 45.7 & 13.5 \\
\hline C.S. Urbano & 293 & 210 & 71.6 & 37.8 & 9.5 \\
\hline Evaluación & 135 & 96 & 71.1 & 38.8 & 9.3 \\
\hline Intervención & 158 & 114 & 72.1 & 37 & 9.7 \\
\hline C. Rural & 191 & 182 & 95.2 & 42.4 & 14.6 \\
\hline Evaluación & 94 & 90 & 95.7 & 42.1 & 15.2 \\
\hline Intervención & 97 & 92 & 94.8 & 42.8 & 14.1 \\
\hline S.M. Empresa & 174 & 152 & 87.3 & 44.9 & 5.7 \\
\hline Evaluación & 74 & 59 & 79.7 & 45.9 & 5.1 \\
\hline Intervención & 100 & 93 & 93 & 44.4 & 6.3 \\
\hline Globales & 1022 & 827 & 80.9 & 42.4 & 12.2 \\
\hline Evaluación & 430 & 346 & 80.4 & $41 ., 9$ & 12.4 \\
\hline Intervención & 592 & 481 & 81.2 & 42.8 & 12.1 \\
\hline
\end{tabular}


En el tabla 1 se presentan los tamaños muestrales iniciales y definitivos en cada contexto estudiado, teniendo en cuenta que en la empresa se trabajó con toda la población de la fábrica aprovechando la revisión médica anual.

\section{Instrumentos}

Se diseñó un protocolo de intervención breve que constaba de un pretratamiento y una evaluación postintervención. Tanto la evaluación pretratamiento como la realizada postintervención fueron efectuadas por procedimiento de autoinforme a través de cuestionarios heteroaplicados preparados por nosotros para la ocasión.

La información recogida en el pretratamiento abarcaba aspectos relativos a las características sociodemográficas y laborales del sujeto, una historia básica de comportamientos de salud en el año anterior y un exhaustivo examen de su historia, problemas asociados, necesidades de atención y patrón habitual de consumo de bebidas alcohólicas en el momento de la entrevista.

En el cuestionario postintervención se recogían posibles cambios en el status sociolaboral, se repetía el examen de la frecuencia y cantidad del consumo habitual de bebidas alcohólicas en ese momento, posibles PRA, y se incorporaba la evaluación de los siguientes aspectos:

- Acontecimientos estresantes en el período

- Autopercepción de relación con el consumo de bebidas alcohólicas

- Autopercepción de efectos sobre el tipo/cantidad de consumo

- Conciencia subjetiva de cambios en el consumo: motivos

- Comportamientos y estrategias utilizados para la disminución

También se utilizó en la primera evaluación el test AUDIT (Babor y cols. 1989), sección no clínica, y el MALT-subjetivo en la versión española de Rodríguez Martos (1984). En el marco del servicio médico de empresa y en el grupo hospitalario se valoró también la parte clínica del AUDIT, así como el MALT-objetivo en el segundo caso.

\section{Diseño}

En todos los ámbitos se utilizó un diseño de dos grupos con asignación aleatoria de los sujetos a las modalidades simple evaluación vs. evaluación más intervención, con registro del consumo pre y postratamiento a los 6 meses de aquel (12 meses en el caso de la empresa). Como aspecto a valorar se consideró el patrón de consumo habitual en unidades/semana tomando como referencia el realizado en la semana anterior a la entrevista y registrando separadamente el efectuado en los cuatro primeros días (lunes a jueves) y el del fin de semana (viernes a domingo). Adicionalmente se planteó también valorar la existencia de posibles PRA asociados evaluando la presencia/ausencia de cada uno en un listado de problemas familiares, legales, sociales, laborales y físicos.

En cuanto al tratamiento, la modalidad simple evaluación consistió en pasar el cuestionario pretratamiento completo. La intervención consistió en añadir a la misma sesión evaluativa, como única ocasión, la entrega de un documento de autoayuda y sobre la explicación del mismo orientar al sujeto en torno su consumo, riesgos y posibles decisiones a tomar.

\section{Procedimiento}

La fuente de casos poblacional fue distinta en cada contexto sociosanitario en que se realizó el proyecto. En el ámbito hospitalario se utilizaron las listas de nuevos ingresos que la administración del centro enviaba diariamente a cada servicio. Después de descartar a quienes por razones de edad o problemática médica no reunían los requisitos de inclusión se seleccionaban diez casos al azar del resto de la lista acudiendo un miembro del equipo investigador a la planta en que se encontraba ingresado cada uno de ellos (máximo de los tres días siguientes a su ingreso), a fin de cumplimentar los datos introductorios y valorar su posible entrada en el estudio. En el ámbito de la atención primaria de salud se eligieron cada día en torno a $2 / 3$ sujetos, escogidos entre los pacientes o acompañantes que estaban esperando ser atendidos por el médico. En la comunidad se utilizó el contacto telefónico, o domiciliario directo, después de seleccionar al azar a los sujetos potenciales de las listas censales. En la empresa, para establecer los criterios de inclusión, fue valorada toda la población de trabajadores entre 18 y 55 años, en la rutina anual de revisión médica.

La forma de seleccionar a los sujetos de estudio fue similar en todos los supuestos: después de cumplimentar la parte del cuestionario pretratamiento correspondiente a las características sociodemográficas, laborales y de consumo, fueron escogidos aquellos varones que reunían los requisitos de edad y consumo ya reseñados. Para delimitar la manera de beber habitual utilizamos un procedimiento de autoinforme ya experimentado por nosotros en estudios previos como el de la Respuesta Comunitaria a los problemas relacionados con el consumo de alcohol (Díez Manrique y Peña Martín, 1989). Por una parte se estimaba la frecuencia mediante una escala ordinal entre la abstinencia y el consumo diario. Por otra parte se valoraba la cantidad, tomando como referencia la semana anterior a la entrevista, a través de un análisis 
detallado, día a día, de los tipos de bebidas (agrupados en seis modalidades según su graduación alcohólica) y de las dosis consumidas de cada una.

En la misma sesión de evaluación pretratamiento, si el sujeto reunía los requisitos de inclusión en el estudio se completaba la toma de datos y era destinado al grupo simple evaluación o intervención. Para ello se utilizó la asignación al azar, por orden de incorporación al estudio, controlando únicamente la edad del sujeto (se trató de equiparar a los grupos en edad apareando los casos con un máximo de 2/3 años de diferencia).

Los sujetos del grupo simple evaluación, una vez cumplimentado el cuestionario pretratamiento, no recibían ningún otro tipo de información o comentario sobre los datos cerrándose en ese momento la situación de entrevista. Los sujetos de intervención recibían en ese mismo momento el folleto de autoayuda y a través de su explicación eran informados sobre su propio consumo, forma de autoevaluarlo, posibles consecuencias sobre de salud y estilo de vida, ideas erróneas y mitos en torno al beber y pautas de comportamientos a tenor del propio consumo. Unos 10 minutos duraba la sesión de evaluación y un tiempo similar la intervención.

A los seis meses de realizada la sesión de evaluación/intervencion se intentó contactar de nuevo con todos los sujetos que habían sido incluidos en el estudio a través del teléfono, carta o directamente visita domiciliaria. Todos los que pudieron ser localizados y accedieron a la entrevista fueron evaluados con el cuestionario de seguimiento, tomando como referencia la situación en ese momento.

\section{Análisis estadístico}

Para el análisis de los datos, nos hemos planteado la comparación entre las evaluaciones pre/post en parámetros como promedio general de consumo, pre- valencia de casos con consumos de riesgo y tasa de frecuencias de sujetos que autorefieren PRA. Hemos utilizado técnicas de contraste para datos relacionados cuando se comparaban cambios intrasujetos o un mismo grupo intercontroles; y técnicas para grupos independientes para los contrastes entre las dos formas de actuación (IB/SE) o entre los contextos. En todos los análisis matemáticos se manejó, como medida del consumo, la cantidad de unidades semanales, según la equivalencia: unidad $=10$ c.c. $=8$ grms. de alcohol

\section{RESULTADOS}

En torno al $20 \%$ del grupo inicial o bien no pudo ser evaluado en el seguimiento o bien tuvo que ser anulado para el análisis por problemas en la recogida y/o codificación de los datos. Las estrategias de localización utilizadas fueron lo suficientemente amplias como para poder afirmar que cuando un caso fue anulado, realmente resultaba totalmente inaccesible su evaluación.

En la tabla 2 se recogen los motivos de perdida de casos situando en el cuerpo de la misma el \% en relación al total del propio contexto. Se consideró como ausente del domicilio cuando no resultaba viable evaluar al sujeto en el mes siguiente a la fecha de entrevista prevista. Entendemos por rechazo aquellas ocasiones en que después de un mínimo de tres contactos personales o telefónicos persistía una actitud de seguir aplazando el encuentro o abiertamente negativa a la evaluación. La anulación técnica se produjo en aquellas situaciones en que existieron problemas de codificación o registro de datos, entre ellos varios casos con valores de consumo previo fuera del rango de inclusión.

Tabla 2. Motivo de perdida de casos en cada contexto ( $\%$ de sujetos del propio grupo).

\begin{tabular}{|c|c|c|c|c|c|c|}
\hline & & \multicolumn{4}{|c|}{ Contexto } & \multirow{4}{*}{$\begin{array}{c}\text { Globales } \\
195\end{array}$} \\
\hline & \multirow[b]{3}{*}{ n perdidas } & \multicolumn{2}{|c|}{ C.S. } & \multirow[b]{2}{*}{ C.Rural } & \multirow{3}{*}{$\begin{array}{l}\text { S.M. } \\
\text { Empresa } \\
22\end{array}$} & \\
\hline & & Hospital & Urbano & & & \\
\hline & & 81 & 83 & 9 & & \\
\hline No localizable & 34 & 13,5 & 25,0 & 33,0 & & 17,4 \\
\hline Ausente & 9 & 9,8 & 1,2 & & & 4,6 \\
\hline Rechazo & 101 & 48,1 & 64,2 & 44,0 & 9,0 & 51,7 \\
\hline Defunción & 8 & 7,4 & 1,2 & & 4,5 & 4,1 \\
\hline Otros & 24 & 12,3 & 1,2 & & 59,1 & 12,3 \\
\hline Anulación técnica & 19 & 8,8 & 7,2 & 22,0 & 27,2 & 9,7 \\
\hline
\end{tabular}


Consumos previos: equivalencia entre los grupos de investigación.

Los resultados obtenidos en la evaluación de la cantidad habitual de consumo, tanto en el control pretratamiento como en el efectuado a los seis meses del mismo (12 meses en la empresa), se recogen en la tabla 3. Se muestran los estadísticos más significativos, así como la relación entre ambos controles en lo referente a diferencia de medias pre/post, y \% de descenso que el consumo postintervención significa del previo.

Tabla 3: Resultados generales de consumo pre y postratamiento y $\%$ medio de descenso

\begin{tabular}{|c|c|c|c|c|c|c|c|c|c|}
\hline & \multicolumn{4}{|c|}{ Preintervención } & \multicolumn{3}{|c|}{ Postintervención } & \multirow{3}{*}{$\begin{array}{l}\text { Dif. } \\
\text { Med. }\end{array}$} & \multirow{3}{*}{$\begin{array}{c}\% \\
\text { menor }\end{array}$} \\
\hline & & & S.E & & & S.E & & & \\
\hline & $\mathrm{N}$ & Media & med. & D.s & Media & med. & D.s & & \\
\hline \multirow{3}{*}{$\begin{array}{l}\text { Hospital } \\
\text { Evaluación } \\
\text { Intervención }\end{array}$} & 283 & 48.60 & 1.19 & 20.1 & 32.43 & 1.78 & 30.1 & 16,1 & 33,2 \\
\hline & 101 & 47.35 & 2.09 & 21.1 & 31.62 & 2.60 & 26.2 & 15.7 & 33.2 \\
\hline & 182 & 49.31 & 1.45 & 19.6 & 32.87 & 2.37 & 32.1 & 16.4 & 33.3 \\
\hline \multirow{3}{*}{$\begin{array}{l}\text { CS. Urbano } \\
\text { Evaluación } \\
\text { Intervención }\end{array}$} & 210 & 48.26 & 1.30 & 18.9 & 36.45 & 1.55 & 22.5 & 11.8 & 24,4 \\
\hline & 96 & 49.92 & 1.96 & 19.2 & 40.03 & 2.15 & 21.1 & 9.9 & 19,8 \\
\hline & 114 & 46.86 & 1.74 & 18.6 & 33.43 & 2.18 & 23.3 & 13.4 & 28,6 \\
\hline \multirow{3}{*}{$\begin{array}{l}\text { C. Rural } \\
\text { Evaluación } \\
\text { Intervención }\end{array}$} & 182 & 43.11 & 1.30 & 17.6 & 38.18 & 1.61 & 21.8 & 4.9 & 11,4 \\
\hline & 90 & 40.00 & 1.63 & 15.5 & 35.51 & 2.08 & 19.8 & 4.5 & 11,2 \\
\hline & 92 & 46.16 & 1.98 & 19.1 & 40.79 & 2.44 & 23.5 & 5.4 & 11,6 \\
\hline \multirow{3}{*}{$\begin{array}{l}\text { SM Empresa } \\
\text { Evaluación } \\
\text { Intervención }\end{array}$} & 152 & 47.19 & 1.44 & 17.8 & 36.52 & 1.44 & 17.7 & 10.7 & 22,6 \\
\hline & 59 & 43.47 & 2.37 & 18.3 & 36.05 & 2.63 & 20.2 & 7.3 & 17,1 \\
\hline & 93 & 49.55 & 1.79 & 17.3 & 36.82 & 1.67 & 16.1 & 12.7 & 25,6 \\
\hline \multirow{3}{*}{$\begin{array}{l}\text { Globales } \\
\text { Evaluación } \\
\text { Intervención }\end{array}$} & 827 & 47.05 & 0.66 & 18.9 & 35.47 & .85 & 24.5 & 11.6 & 24,6 \\
\hline & 346 & 45.49 & 1.02 & 19.1 & 35.72 & 1.2 & 22.4 & 9.8 & 21,4 \\
\hline & 481 & 48.18 & 0.85 & 18.8 & 35.28 & 1.1 & 26.1 & 12.9 & 26,7 \\
\hline
\end{tabular}

Puede plantearse como primera aproximación a estos datos en qué medida son similares las poblaciones de origen en sus cantidades de consumo. De forma global se aprecian diferencias consistentes en menor consumo por parte de los sujetos de la comunidad rural, que alcanzan significación estadística (ANOVA de una vía, Oneway, gr.l=3, $F=3.58, p<.05$, prueba de Scheffé) en relación al grupo hospitalario. Un análisis más detallado, comparando por separado el consumo previo de todos los grupos asignados a cada modalidad de actuación (simple evaluación / intervención breve), permite observar que así como en los sujetos de intervención no existen diferencias de consumo intercontextuales (Oneway, $F=.92$, gr.l. = $3, p>.05)$, por el contrario los sujetos de evaluación en la comunidad rural de nuevo aparecen como bebedores de menor cantidad que el resto de la misma condición en los otros ámbitos $(F=4.92$, gr.l. $=3, p<.01$, prueba de Scheffé, diferencia entre el grupo rural y el del centro de salud)

Al comparar los consumos previos de todos los sujetos asignados a evaluación vs. intervención se observan diferencias estadísticamente significativas $(t=2.01, p<.05)$ consistentes en menor consumo por parte del grupo de evaluación y provocadas por el efecto que tiene sobre el total la presencia de esa relación en la comunidad rural y en la empresa $(t=2.39$ y 2.09 respectivamente, $p<.05$ en ambos casos).

Parece poder concluirse en consecuencia que, junto a otras posibles condiciones no controladas, los efectos de azar han generado grupos de investigación algo diferentes en sus consumos previos. Esta condición no tiene porqué afectar especialmente al análisis de datos, en la medida en que para profundizar en los cambios pre/post cada individuo funciona como su propio control, y para las comparaciones intergrupos 
decidimos trabajar con las puntuaciones diferenciales de cada sujeto.

Por otra parte junto al sesgo de representatividad poblacional generado por los sujetos incluidos en el estudio (rango de 21 a 95 unidades) parecen existir factores, asociados a la fuente contextual de casos, sugerentes de que los sujetos tomados directamente de la comunidad presentan un patrón de consumo de menor cantidad que los conectados en los diversos entornos sanitarios.

\section{Tabla 4: Resultados de la comparación de consumos pre-post intergrupos (Test de Wilcoxon)}

\begin{tabular}{|ccccc|}
\hline & \multicolumn{3}{c}{ Media de rangos } & Z \\
\cline { 2 - 5 } Hospital & $\mathrm{N}$ & Pre. & Post. & \\
Evaluación & 283 & 144.6 & 124.4 & -8.67 \\
Intervención & 101 & 53.6 & 40.1 & -5.50 \\
CS. Urbano & 182 & 91.5 & 84.6 & -6.73 \\
Evaluación & 210 & 112.9 & 75.2 & -7.64 \\
Intervención & 96 & 51.4 & 38.3 & -4.37 \\
C. Rural & 114 & 61.9 & 36.8 & -6.33 \\
Evaluación & 182 & 98.3 & 63.7 & -4.77 \\
Intervención & 90 & 47.6 & 29.0 & -2.75 \\
SM Empresa & 92 & 50.8 & 36.6 & -3.79 \\
Evaluación & 152 & 77.2 & 51.7 & -7.35 \\
Intervención & 59 & 28.3 & 24.8 & -3.61 \\
Globales & 93 & 48.9 & 27.3 & -6.40 \\
Evaluación & 827 & 433.5 & 308.3 & -14.37 \\
Intervención & 346 & 180.4 & 124.3 & -8.50 \\
& 481 & 251.3 & 185.6 & -11.67 \\
\hline
\end{tabular}

$Z$ con $\mathrm{p}<.001$ en todos los casos

\section{Cambios globales en el consumo pre-post}

Como puede observarse en la tabla 4 se produce en todos los contextos y grupos estudiados un descenso estadísticamente significativo en la cantidad habitual semanal de consumo de bebidas alcohólicas. En términos cuantitativos (tabla 3 ) este descenso significa globalmente 11,6 u./semana menos de consumo con un rango que va de las $4.5 \mathrm{u}$. que reducen los sujetos del grupo evaluación en la comunidad rural a las $16.4 \mathrm{u}$. en el grupo de intervención hospitalaria. Dicho de otra forma, los consumos promedios, a los seis meses de la primera evaluación, significan un global del $24.6 \%$ menos que en aquella, con un rango entre el 11 y el 33 \% (evaluación en comunidad rural e intervención en el grupo del hospital respectivamente).

Si bien parece clara la presencia global de menores consumos postintervención que los existentes en la evaluación previa, podría darse el caso de que la cuantía de dicho descenso no tuviese relevancia clínica o también la existencia de efectos de azar que no permitieran sugerir una tendencia en los efectos. Cuando los resultados se sometieron a la prueba de McNemar para la significación de los cambios se pudo observar que cuando los sujetos habían modificado su intervalo de consumo lo hacían, de una forma significativa en todos los casos, hacia niveles más reducidos.

\section{Cambios pre/post en la prevalencia de bebedo- res de riesgo}

Dado que uno de los objetivos del proyecto consistía en lograr consumos más controlados y moderados, y una forma de operativizar el mismo consistió en establecer niveles de mayor/menor peligrosidad, cabe preguntarse en qué medida se producen cambios en la prevalencia de consumidores de riesgo, entendiendo por tales aquellos que toman una cantidad igual o superior a las 35 u./semana. El estudio de los resultados de la tabla 5 , donde se recogen las prevalencias pre/post de bebedores excesivos así como la significación de diferencias entre ambas, permite afirmar la 
existencia de un beneficio generalizado a la mayoría de contextos/grupos, consistente en la notable y significativa disminución del numero de personas con consumos de riesgo, de forma que la prevalencia glo- bal desciende en un 17\% e incluso, cuando el cambio no es significativo, grupo de evaluación en la comunidad rural, existe en torno a un $10 \%$ menos de bebedores de riesgo

Tabla 5: Cambios en la prevalencia de bebedores de riesgo

\begin{tabular}{|c|c|c|c|c|c|c|c|}
\hline & & \multicolumn{2}{|c|}{ Preintervención } & \multicolumn{2}{|c|}{ Postintervención } & \multirow[t]{2}{*}{$\%$ menor } & \multirow[b]{2}{*}{ Rc } \\
\hline & $\mathrm{N}$ & $n$ & Prevalencia & $n$ & Prevalencia & & \\
\hline Hospital & 283 & 177 & 62.54 & 107 & 37.80 & 24.7 & $9.59 * *$ \\
\hline Evaluación & 101 & 57 & 56.43 & 41 & 40.59 & 15.8 & $3.47 * *$ \\
\hline Intervención & 182 & 120 & 65.93 & 66 & 36.26 & 29.7 & $9.54 * *$ \\
\hline CS. Urbano & 210 & 137 & 65.23 & 104 & 49.52 & 15.7 & $4.96^{* *}$ \\
\hline Evaluación & 96 & 69 & 71.87 & 55 & 57.29 & 14.6 & $3.09 * *$ \\
\hline Intervención & 114 & 68 & 59.64 & 49 & 42.98 & 16.7 & $3.89 * *$ \\
\hline C. Rural & 182 & 104 & 57.14 & 86 & 47.25 & 9.9 & $2.81^{*}$ \\
\hline Evaluación & 90 & 46 & 51.11 & 39 & 43.33 & 7.8 & 1.53 n.s \\
\hline Intervención & 92 & 58 & 63.04 & 47 & 51.08 & 11.9 & $2.44^{*}$ \\
\hline SM Empresa & 152 & 98 & 64.47 & 70 & 46.05 & 18.4 & $5.02 * *$ \\
\hline Evaluación & 59 & 31 & 52.54 & 22 & 37.28 & 15.3 & $2.54^{*}$ \\
\hline Intervención & 93 & 67 & 72.04 & 48 & 51.61 & 20.4 & $4.41 * *$ \\
\hline Globales & 827 & 516 & 62.31 & 367 & 44.32 & 17.9 & $11.43 * *$ \\
\hline Evaluación & 346 & 203 & 58.67 & 157 & 45.37 & 13.3 & $5.31 * *$ \\
\hline Intervención & 481 & 313 & 65.07 & 210 & 43.65 & 21.4 & $10.59 * *$ \\
\hline
\end{tabular}

Cambios en el consumo post de los bebedores iniciales de riesgo

En muchos de los estudios que hemos revisado los destinatarios del plan de acción solían ser bebedores de cantidades altas o "de riesgo", estableciendo en muchos casos la línea de corte en 280 gr./semana (34/35 u. semanales). Al haber incorporado nosotros bebedores más moderados, parece pertinente analizar específicamente cuál es el comportamiento postintervención de los sujetos que eran bebedores de riesgo. En concreto, en qué medida se benefician del programa estableciendo como criterio para ello que modifiquen su nivel de consumo previo por debajo de las 35 u./280 gr. semanales. En la tabla 6 exponemos el análisis de estos datos.

Tabla 6: Cambios en nivel de consumos de los bebedores iniciales de riesgo

\begin{tabular}{|c|c|c|c|c|c|c|c|}
\hline \multirow{5}{*}{$\begin{array}{l}\text { Hospital } \\
\text { Evaluación } \\
\text { Intervención }\end{array}$} & \multirow{2}{*}{$\mathrm{N}$} & \multicolumn{4}{|c|}{ consumos postratamiento (34/35 u.) } & \multirow{2}{*}{ E.Max } & \multirow{2}{*}{$\mathrm{Rc}^{*}$} \\
\hline & & \multicolumn{2}{|c|}{ menor } & \multicolumn{2}{|c|}{ igual } & & \\
\hline & 177 & 101 & 57.1 & 76 & 42.9 & .07 & 15.3 \\
\hline & 57 & 31 & 54.4 & 26 & 45.6 & .12 & 8.2 \\
\hline & 120 & 70 & 58.3 & 50 & 41.7 & .08 & 12.9 \\
\hline CS. Urbano & 137 & 52 & 38.0 & 85 & 62.0 & .08 & 9.2 \\
\hline Evaluación & 69 & 23 & 33.3 & 46 & 66.7 & .11 & 5.9 \\
\hline Intervención & 68 & 29 & 42.6 & 39 & 57.4 & .11 & 7.1 \\
\hline C. Rural & 104 & 29 & 27.9 & 75 & 72.1 & .08 & 6.3 \\
\hline Evaluación & 46 & 12 & 26.1 & 34 & 73.9 & .12 & 4.0 \\
\hline Intervención & 58 & 17 & 29.3 & 41 & 70.7 & .11 & 4.9 \\
\hline SM Empresa & 98 & 36 & 36.7 & 62 & 63.3 & .09 & 7.5 \\
\hline Evaluación & 31 & 14 & 45.2 & 17 & 54.8 & .17 & 5.0 \\
\hline Intervención & 67 & 22 & 32.8 & 45 & 67.2 & .11 & 5.7 \\
\hline Globales & 516 & 218 & 42.2 & 298 & 57.6 & .04 & 19.4 \\
\hline Evaluación & 203 & 80 & 39.4 & 123 & 60.6 & .06 & 11.5 \\
\hline Intervención & 313 & 138 & 44.1 & 175 & 55.9 & .05 & 15.7 \\
\hline
\end{tabular}

*: Razón critica con $\mathrm{p}<.01$ en todos los casos 
Tomando como referencia los sujetos que en cada contexto/grupo tomaban esa cantidad (516 casos, $62 \%$ del total), se presenta en la tabla el numero y $\%$ de casos que mantiene o disminuye su intervalo de consumo previo, el error máximo de estimación y la significación de ese descenso en cada caso. El error máximo de estimación se ha calculado sobre un nivel de riesgo del $5 \%$, y la significación del cambio, en el porcentaje de casos bebedores altos, se ha calculado sobre la hipótesis de compatibilidad de la nueva prevalencia con la situación previa donde todos ellos cumplían esa condición $(p=1)$. Observando los resultados parece confirmarse, también en este caso, el impacto del programa de actuación al considerar el importante numero de sujetos bebedores altos que al ser reevaluados pudo objetivarse que habían modificado su nivel de consumo. Puede afirmarse que existe un porcentaje de sujetos, estadísticamente significativo en todas las condiciones analizadas, que parece habían moderado su consumo habitual situándolo en cantidades de menor riesgo. Las cifras fluctúan entre un mínimo del $26 \%$ en el grupo de evaluación en la comunidad rural hasta un 58\% en el grupo de intervención hospitalaria, si bien en algunos casos los pequeños tamaños muestrales generan un error de estimación suficientemente amplio como para interpretar cautelosamente las magnitudes porcentuales. De todas formas puede sugerirse que, en general, en torno al $42 \%$ de los primitivos bebedores de riesgo se beneficiaron del programa de actuación en la medida en que sus consumos post se situaban en niveles más controlados y asumibles.

\section{Efectos asociados a la simple evaluación vs intervencion}

Por lo expuesto hasta este momento, se ha podido constatar efectos principales en todos los indicadores de consumo utilizados (cantidad promedio semanal, prevalencia de bebedores de riesgo y proporción de estos que descienden a niveles más moderados), tanto en los sujetos que simplemente fueron evaluados como en aquellos que además fueron intervenidos. La cuantía de los cambios, siempre en la dirección esperada de mejorías post frente a niveles previos, es estadísticamente significativa en la practica totalidad de las ocasiones. El efecto generalizado de cambio en el consumo cuestiona, en consecuencia, la posible hipótesis según la cual, considerando el grupo de simple evaluación como un grupo de control no tratado, debería mantener en la reevaluación el mismo estilo de bebida que tenía al inicio.

Un análisis más profundo de los efectos diferenciales asociados a la modalidad de accion (SE / IB), Io hemos abordado desde enfoques complementarios que tratan de responder a interrogantes sobre los cambios en la cantidad promedio de consumo semanal, los cambios en la prevalencia de bebedores de riesgo, y el descenso a consumos más moderados por parte de quienes inicialmente eran bebedores altos. En la tabla 7 se recogen los estadísticos resultantes de cada contraste entre sujetos evaluados vs intervenidos.

Para el estudio de la cantidad de consumo hemos utilizado la prueba no paramétrica de dos muestras independientes de Mann-Whitney manejando como variable dependiente las unidades diferenciales de consumo pre-pos. Existe una diferencia de promedios pre/post siempre superior en el grupo intervención. que es variable según el contexto de estudio, mínima en el entorno hospitalario y de la comunidad rural (en torno a $1 \mathrm{u}$./promedio semanal) y máxima en la actuación en la empresa (5 u./semana a los 12 meses). Solo se alcanzan valores de significación estadística en el marco del servicio médico de empresa $(z=2.08)$ y al comparar todos los sujetos ( $z=2.89$ ) como consecuencia de la tendencia general.

Tabla 7: Análisis de las diferencias intergrupos: simple evaluación vs intervención.

\begin{tabular}{|c|c|c|c|c|c|c|c|c|c|}
\hline & \multicolumn{3}{|c|}{$\begin{array}{c}\text { Cambios en la cantidad } \\
\text { de consumo } \\
\text { (Test de Mann Whitney) }\end{array}$} & \multicolumn{3}{|c|}{$\begin{array}{l}\text { Prevalencia de bebedores } \\
\text { de riesgo .Cambio pre/post } \\
\text { (Razón critica) }\end{array}$} & \multicolumn{3}{|c|}{$\begin{array}{l}\text { Descenso de nivel de } \\
\text { los bebedores de } \\
\text { riesgo }(n=516) \mathrm{Chi}^{2}\end{array}$} \\
\hline & $\mathrm{N}$ & $\begin{array}{c}\text { Md. } \\
\text { Rango }\end{array}$ & z & Pre & Post & z & $\mathrm{n} 1$ & $\mathrm{n} 2$ & $\mathrm{Chi}^{2}$ \\
\hline $\begin{array}{l}\text { Hospital } \\
\text { evaluación } \\
\text { intervención }\end{array}$ & $\begin{array}{l}283 \\
101 \\
182 \\
\end{array}$ & $\begin{array}{r}137.1 \\
144.6 \\
\end{array}$ & .73 & 9.41 & 4.33 & $\begin{array}{r}1.58 \\
.72 \\
\end{array}$ & $\begin{array}{r}57 \\
120 \\
\end{array}$ & $\begin{array}{l}26 \\
50 \\
\end{array}$ & .24 \\
\hline $\begin{array}{l}\text { C.S. Urbano } \\
\text { evaluación } \\
\text { intervención }\end{array}$ & $\begin{array}{r}210 \\
96 \\
114\end{array}$ & $\begin{array}{r}98.9 \\
111.1\end{array}$ & 1.43 & 12.23 & 14.31 & $\begin{array}{l}1.85 \\
2.07^{*}\end{array}$ & $\begin{array}{l}69 \\
68\end{array}$ & $\begin{array}{l}46 \\
39\end{array}$ & 1.26 \\
\hline $\begin{array}{l}\text { C. Rural } \\
\quad \text { evaluación } \\
\text { intervención }\end{array}$ & $\begin{array}{r}182 \\
90 \\
92\end{array}$ & $\begin{array}{l}86.1 \\
96.8 \\
\end{array}$ & 1.36 & 11.93 & 7.76 & $\begin{array}{l}1.63 \\
1.05\end{array}$ & $\begin{array}{l}46 \\
58 \\
\end{array}$ & $\begin{array}{l}34 \\
41\end{array}$ & .13 \\
\hline $\begin{array}{l}\text { S.M.Empresa } \\
\text { evaluación } \\
\text { intervención }\end{array}$ & $\begin{array}{r}152 \\
59 \\
93\end{array}$ & $\begin{array}{l}67.2 \\
82.4\end{array}$ & $2.08^{*}$ & 19.50 & 14.32 & $\begin{array}{r}2.45^{*} \\
1.73\end{array}$ & $\begin{array}{l}31 \\
67\end{array}$ & $\begin{array}{l}17 \\
45\end{array}$ & 1.38 \\
\hline $\begin{array}{l}\text { Globales } \\
\quad \text { evaluación } \\
\text { intervención }\end{array}$ & $\begin{array}{l}827 \\
346 \\
481\end{array}$ & $\begin{array}{l}385.6 \\
434.4\end{array}$ & $2.89 * *$ & 6.40 & 1.72 & $\begin{array}{r}1.87 \\
.49\end{array}$ & $\begin{array}{l}203 \\
313\end{array}$ & $\begin{array}{l}123 \\
175\end{array}$ & 1.10 \\
\hline
\end{tabular}

\footnotetext{
${ }^{*}=p<.05 y^{* *}=p<. .01$
} 
En el estudio de los cambios en las prevalencias de bebedores de riesgo entre los sujetos SE vs IB, se debe tener en cuenta, como se ha señalado con anterioridad, que la asignación aleatoria de los sujetos produjo algunas condiciones según las cuales los grupos de investigación surgidos presentaron características algo distintas en sus consumos previos. En cada contexto de actuación, hemos comparado intergrupos, simple evaluación vs. intervención breve, las prevalencias pre y post por separado. Si se interpretan los resultados de la tabla 7 , teniendo en cuenta las consideraciones previas y los valores de la tabla 5, la ausencia de significación estadística en los contrastes lejos de cuestionar confirmaría la hipótesis de efectos diferenciales asociados al tipo de actuación, con una clara tendencia a mayor descenso en las tasas postratamiento en todos los grupos de intervención frente a los de simple evaluación.

Un ultimo planteamiento tiene que ver con el comportamiento en el consumo post de aquellos sujetos que previamente eran bebedores excesivos, destinatarios específicos, en muchos estudios, de programas como el que aquí presentamos. En qué medida resultaron más beneficiados los sujetos asignados a intervención frente a los que simplemente fueron evaluados, puede responderse si se compara, entre ambos grupos, el numero proporcional de casos que modificaron su nivel previo de 35 o más unidades semanales. Los valores de $\mathrm{Chi}^{2}$ resultantes son muy bajos, en ninguno caso alcanzan significación estadística y, en consecuencia, permiten afirmar que, en cuanto al numero de casos que modifican un consumo previo de riesgo, los efectos de la simple evaluación fueron similares a los obtenidos con la intervención-consejo.

\section{DISCUSIÓN}

Las características de consumos previos encontradas en los sujetos de estudio son básicamente coincidentes, tanto en tasa de bebedores habituales como en cantidad y forma de distribución del consumo, con los resultados de otros estudios realizados por nosotros (Díez Manrique, 1991). Es de señalar la similitud de promedios de consumo semanal referida por los sujetos de los distintos contextos, que indirectamente sugiere la validez del procedimiento al no existir razones para hipótesis de diferencias previas. Incluso el menor consumo objetivado en los sujetos de la comunidad rural frente a los del hospital, podría estar apuntando un aspecto constatado en la investigación epidemiológica y consistente en la alta prevalencia de PRA presente en las consultas-ingresos de hospital general especialmente medicina interna y digestivo (Solé Puig y Freixa, 1988)

Una primera aproximación a los resultados nos permite concluir que el programa diseñado, al menos a corto plazo, parece haber influido sobre los sujetos de estudio de forma que hemos podido constatar cambios significativos hacia le mejoría en todos los parámetros de medida que hemos utilizado. Un 55\% de casos tomaba como promedio semanal al menos 7 u. menos que al principio; el promedio de consumo había descendido en 11.6 u./semana, que significaba un $25 \%$ del inicial; la prevalencia de bebedores de riesgo había descendido en un $11 \%$; y un $42 \%$ de quienes inicialmente lo era habían moderado su consumo hacia umbrales inferiores a ese nivel. Estos resultados son muy similares en los aspectos "comparables" a los que se informan en estudios como el de Wallace y cols.(1988) o el multinacional de la OMS (Babor y Grant, 1992), quienes manejan indicadores parcialmente similares a los utilizados por nosotros.

Un efecto teóricamente inesperado es el que se produce en los sujetos del grupo simple evaluación en la medida en que también en este caso se evidencian cambios pre-post intragrupo, estadísticamente significativos, en la mayoría de indicadores de consumo que hemos utilizado y prácticamente generalizados a todos los contextos. ¿Cómo explicar la mejoría de los sujetos de control?. Reflexionando sobre el problema que también está presente en los resultados del estudio transcultural de la OMS, Babor (1994) se pregunta si ¿regresión a la media o efecto de la evaluación?. En su caso, considerando las características de la evaluación, plantea que presumiblemente los sujetos de control ignoraban los propósitos del estudio por lo que considera más plausible el conocido efecto de regresión estadística, consistente en una aproximación de valores a los promedios en una reevaluación cuando se trabaja con sujetos de puntuaciones extremas. Por su parte Wallace y cols. (1988) admiten tanto este fenómeno como posibles efectos de la evaluación, al mismo tiempo que consideran que el cambio en los sujetos de control minimiza la verdadera magnitud del efecto de la intervención. Bien y cols (1993) plantean que cuando se están comparando resultados realmente se están comparando procedimientos, y sugieren que una de las posibles razones de ausencia de diferencias entre la intervención breve y las condiciones de control puede deberse a la presencia en los grupos control ("no tratados") de componentes de IB, elementos motivacionales para el cambio o incluso modelos de intervención pobres. La amplia evaluación inicial realizada en nuestro caso, nos lleva a plantear un efecto de reactancia de la evaluación de tal forma que ya en sí misma comportaría elementos de toma de conciencia y posible motivación para el cambio. Una razón similar podría explicar la ausencia de diferencias intergrupos en los sujetos del proyecto DRAMS (Heather y cols., 1987) en el que todos ellos habían recibido evaluación previa de sus problemas de bebida. 
los estudios sobre intervención breve son tan heterogéneos en fuentes de casos y formas de reclutamiento, criterios de inclusión, procedimientos y componentes utilizados, tipo y numero de variables dependientes, formas de medir el consumo etc. que parecería coherente preguntarse ¿cómo contrastar estudios tan dispares que muchas veces solo tienen en común el nombre de intervención breve y un genérico objetivo de incidir sobre el consumo en sujetos bebedores de riesgo?. No se contrastan resultados, se contrastan procedimientos. Si como parece suceder con frecuencia, no se cumplen condiciones mínimas de similitud metodológica, ¿qué sentido tiene este contraste en aras de una buena generalización?. Más aún, el estado actual de conocimientos y, en concreto, los resultados que se desprenden del estudio multinacional de la OMS, permiten afirmar que son estrategias que logran unos efectos modestos pero robustos, consistentes y clínicamente significativos, en sus distintas variantes en todos los settings de salud y en todas las condiciones socioculturales (Kristension y Osterling, 1994). De esta forma se hace irrelevante la pregunta ¿funciona la intervención breve?. Quizá por eso Heather (1994) considera la intervención breve como una "alternativa terapéutica" y no como una submodalidad de otras formas de tratamiento.

¿Porqué son eficaces las estrategias de intervención breve?. Si se utilizan como referente los componentes del modelo FRAMES (Miller y Sánchez, 1993) pocos estudios poseen los seis elementos y quizá el único presente siempre sea el "consejo para el cambio". Por su naturaleza abreviada estos procedimientos tienen pocas o ninguna de las condiciones que los autores especulan como mínimamente necesarias para favorecer el cambio en las conductas aditivas (Miller, 1989; Orford, 1986; Ritson, 1986). Las estrategias de IB han focalizado su atención en la toma de conciencia y consejo para el cambio. ¿será esto suficiente?. Miller y Rollnick, (1991) consideran plausible que el impacto primario de la IB sea sobre la motivación para el cambio y que, una vez producida, los individuos procedan al cambio de conducta con una asistencia adicional mínima, y de hecho los cambios substanciales en el consumo se producen en las primeras semanas después de la intervención. Sea cual sea la eficacia diferencial de los componentes, o sean cuales sean aquellos necesarios, parece poder concluirse que con bajo coste y fácil aplicabilidad las estrategias de intervención breve producen unos resultados que pueden resultar modestos pero fiables y clínicamente relevantes sobre la conducta de beber y problemas relacionados, especialmente con sujetos bebedores no alcohol dependientes. Es buena forma de prevención primaria y secundaria de los problemas relacionados con el consumo de alcohol desde entornos sociosanitarios muy diversos

\section{BIBLIOGRAFÍA}

Babor, T.F. (1994). The WHO brief intervenion study, warts and all. A response to the commentators. En Commentaries: Comments on the WHO report "Brief Interventions for Alcohol Problems":a summary and some international comments. Addiction, 89, 676-678.

Babor, T.F., Fuente de la, J.R., Saunders, J., y Grant M. (1989). AUDIT: The alcohol use disorders identification test. Ginebra: World Health Organization (WHO).

Babor, TF, y Grant, M. (edit.) (1992). Project on identification and management of alcohol-related-problems. Report on Phase II. A randomized clinical trial of brief interventions in primary healt care. Geneva:WHO.

Bien, TH., Miller, WR., , y Tonigan JS. (1993). Brief Interventions for alcohol problems: a review. Addiction, 88, 315336.

Carné, X., Moreno, V., Porta Serra, M., y Velilla, E. (1989). El cálculo del numero de pacientes necesarios en pla planificacion de un estudio clinico. Medicina Clínica, 92, 72-78

Díez Manrique, J.F., y Peña Martín, C. (1989). Problemas relacionados con el consumo de alcohol en Cantabria. Perfiles sociodemográficos de consumo. En J.F. Díez Manrique y J.L. Vazquez Barquero (Coor.): XIV Reunión nacional de la sociedad española de psiquiatría biológica. Madrid: Aran S.A.

Díez Manrique, J.F. (1991). Respuesta comunitaria a los problemas relacionados con el alcohol: Resultados del estudio en Cantabria. En Ministerio de Sanidad y Consumo (Ed.), Respuesta comunitaria a los problemas relacionados con el alcohol. Madrid: Ministerio de Sanidad y Consumo.

Gutierrez Perez A. (1993). Estudio de Variables sociodemográficas que influyen en la aparición de los problemas relcionados con el alcohol (PRA) en Cantabria. Tesis Doctoral. Santander Dpto. de Medicina y Psiquiatría. Facultad de Medicina.

Heather, N. (1994). Brief Interventions on the world map. En Commentaries: Comments in the WHO report "Brief Intervention for Alcohol Problems": a summary and some international comments. Addiction, 89, 665-667

Kristenson, H., y Osterling, A. (1994). Problems and possibilities. Commentaries: Comments on the WHO report "Brief Interventions for Alcohol Problems": a summary and some international comments. Addiction, 89, 671-674

Miller, WR., Sovereign, R., Nathan, PE., y Marlatt, GA. (Edits.) (1989). Addictive Behaviours: Prevention and Early Intervention. WHO.

Miller, W.R. (1989). Raiding the lost ark:do we need new models to study behavior change in spiritual contexts?. Spiritual and Religious Issues in Behavio Change, 4, 615

Miller, W.r., y Rollinick, S. (1991). Motivational Interviewing: Preparing People to Change Addictive Bevavior. New York:Guilford Press. 
Miller, W.R., y Sanchez, V.C. (1993). Motivating young adults for treatment and lifestyle change. En Howard G. (Ed.), Issues inalcohol Use and Misuse by Young Adults. Notre Dame In:University of Notre Dame Press.

Moser, J. (1989). Alcohol problems, policies and programmes in Europa. En WHO (Ed.), Collaborative study on conmunity response to alcohol related problems. San Marino:WHO.

Orford, J. (1986). Critical conditions for change in the addictive behaviors. En W.R. Miller y N. Heather (Edits.), Treating Addictive Behaviors: Procces of Change. New York:Plenum Press.

Peña Martín, C. (1995). Indicadores de Salud. En JF. Díez Manrique (Dir.): Estudio de Salud de Santander. Santander Ayuntamiento de Santander y Universidad de Cantabria.

Ritson, E.B. (1985). Community Response to AlcoholRelated-Problems: Review of an International Study. Geneva WHO. Public Healt Paper, $n^{\circ} 81$.
Ritson, EB. (1986). Research and action: Lesson from the world health organitation project on community response to alcohol-related problems. Annales NY Academics sciencies, 472, 33-45

Rodriguez Martos, A. (1994). Papel de la atencion primaria en el tratamiento de los problemas relacionados con el consumo de alcohol: motivacion para el cambio. Atencion Primaria, 14,4, 743-751

Rodriguez Martos, A. (1989). Manual de alcoholismo para el médico de cabecera. Barcelona:Salvat.

Solé Puig, J.T., y Freixa, F. (1988). Epidemiología del acoholismo en España. En P.A. Soler, F. Freixa y F. Reina (Dirs.), Trastornos por dependencia del alcohol. Barcelona: Delagrange.

Wallace, P., Cutler, S. y Hames, A. (1988). Randomised controlled trial of general practitioner intervention in patiients with excesive alcohol consumption. Britis Medical Journal, 297, 663-668. 